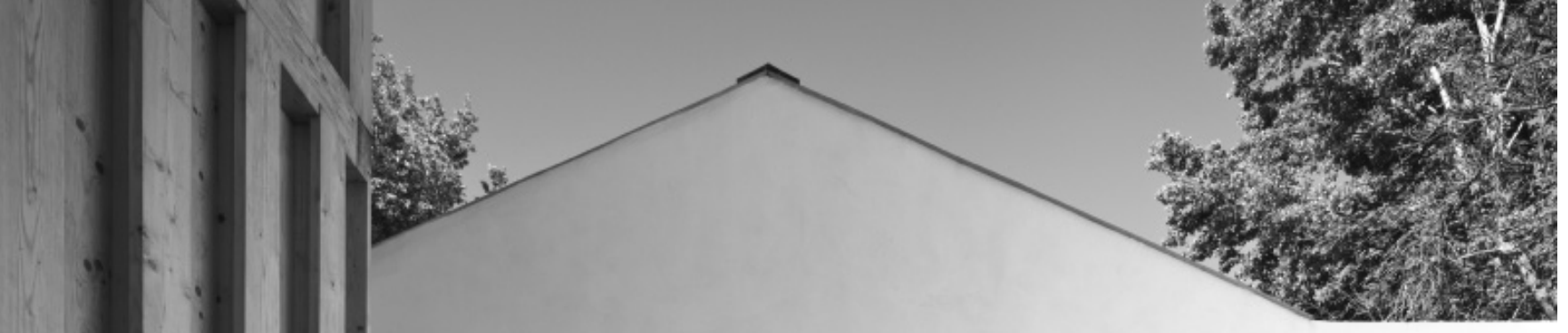

PROYECTIOYYMATIERIAI
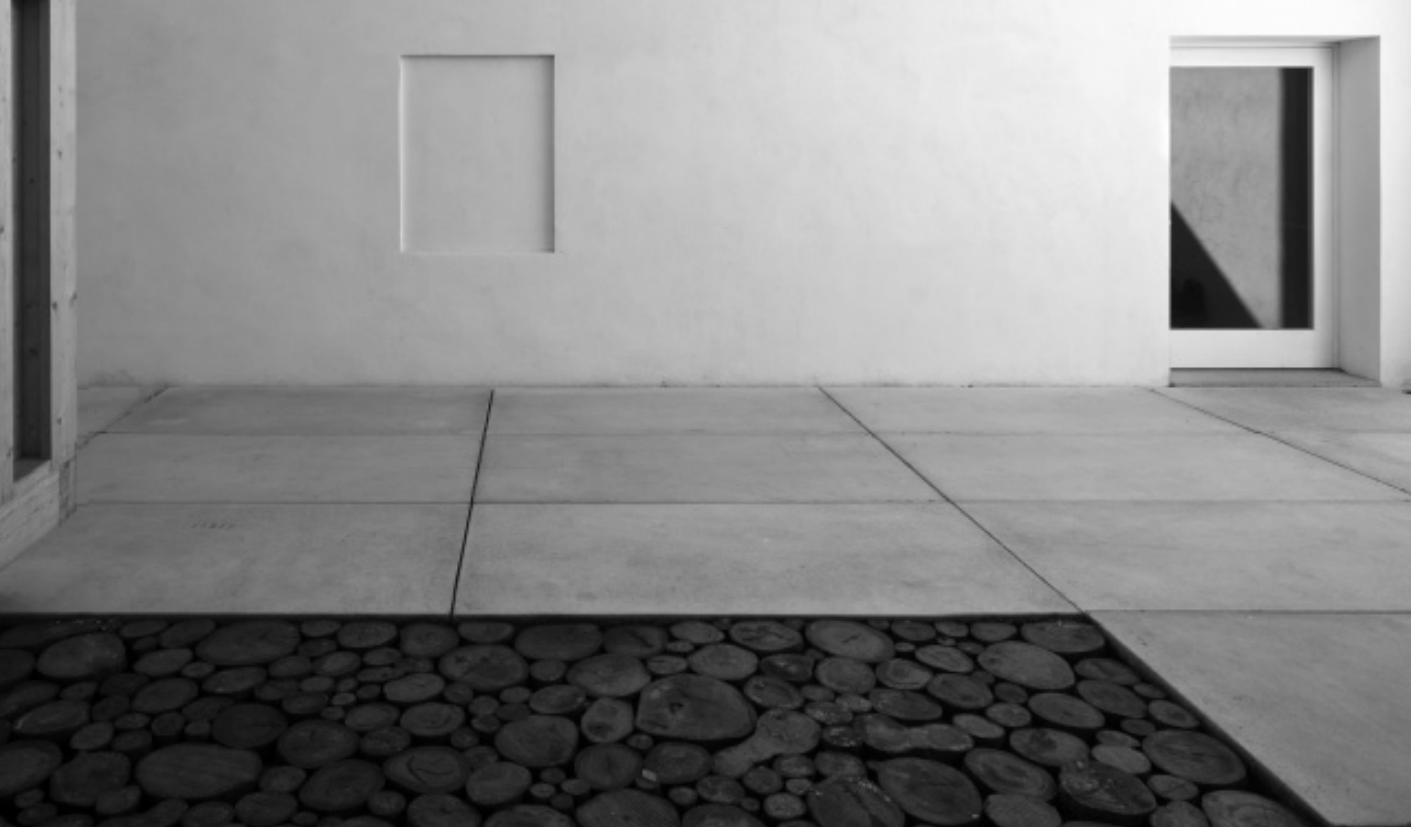

^ ( ) Fernando Alda

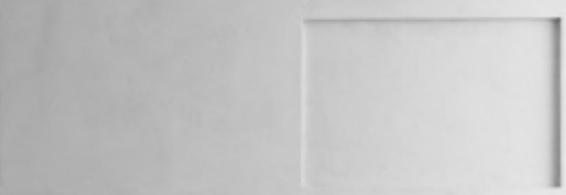

Museo del agua de Lanjarón

Renovación de un antiguo molino de agua

Juan Domingo Santos

Juan Domingo Santos es Doctor Arquitecto y profesor de Proyectos Arquitectónicos en la E.T.S.A. de Granada.

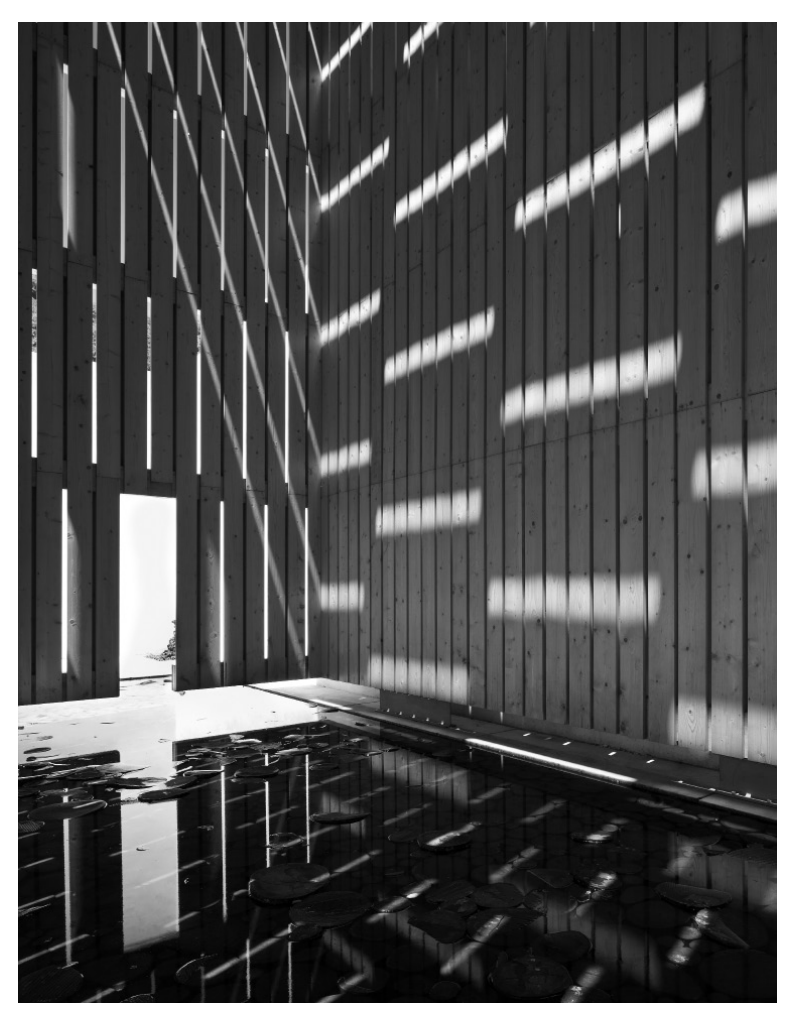

$\wedge$ ( ) Fernando Alda

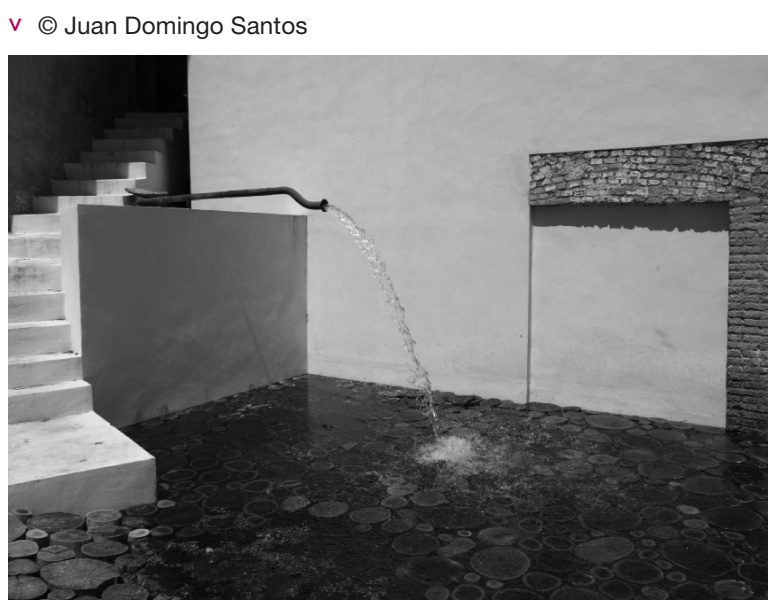

El proyecto del museo se inició con la búsqueda de un lugar donde se favoreciera la presencia del agua en unas condiciones naturales. El espacio elegido se encuentra situado en el acceso al Parque natural de Sierra Nevada, junto al río Lanjarón y una acequia de vada, junto al rio Lanjarony una acequia de
riego que bordea unas antiguas construccioriego utilizadas como matadero municipal.

Dados los escasos medios disponibles la intervención ha consistido en el reciclaje y reutilizacion de algunos elementos del entorno. Las naves del matadero, por ejemplo, se han adaptado a museo, y se han incorporado a las nuevas instalaciones los trazados de agua de la acequia y el río a través de un sencillo sistema de láminas de agua conectadas entre sí. Delante del conjunto se ha dispuesto una plaza de naranjos ligeramente elevada del suelo, con prefabricados de hormigón apilados y troncos de eucalipto de diferentes tamaños que se inundan temporalmente con el agua de la acequia, lo que configura un espacio con aspecto diferente a lo largo del día. La sombra y el olor a azahar de los naranjos, el sonido del agua al caer sobre los 列 .

El ingreso se produce ocupando el patio del antiguo matadero con una nueva construcción en madera. Este pabellón alberga un espacio representativo dedicado al agua y se convierte en un hito de referencia en el paisaje. La construcción evoca la cubrición del Manantial de la Capuchina, una construcción del siglo XVIII realizada en madera que albergaba en su interior el primer nacimiento de agua en Lanjarón. El nuevo pabellón está concebido como un espacio para los sentidos, suspendido en el aire y con dos aperturas que permiten al visitante acceder al interior y participar de los efectos de luz y penumbra. Una lámina de agua extendida sobre el suelo refuerza aún más estas sensaciones, similares a las de los baños islámicos.

La intervención en las antiguas naves ha sido mínima y ha consistido en la demolición de las divisiones interiores, dejando a la vista las estructuras de paredes y cubiertas. Durante los trabajos se pudo descubrir que originalmente la estructura perrecuperación ha adquirido una dimensión arqueológica.

Los espacios expositivos se han dispuesto mediante una ocupación selectiva del interior de las antiguas construcciones, dejando los corrales y otras dependencias inutilizados hasta futuras necesidades. Con el fin de contrastar los muros de piedra y ladrillo del antiguo molino, se han dispuesto de manera localizada paneles trasdosados en color blanco que enmarcan los lugares de la nueva intervención. Las dos naves principales se temática de contenidos. En la nave más antigua un vidrio con proyecciones sobre su superficie emerge del suelo inundado con agua de la acequia, creando un juego de reflejos sobre los antiguos muros del molino.
Recurso ambiental. Uso del agua en el medio natural El museo se estructura en torno a los trazados de agua de una acequia a los que se vinculan tres nuevas láminas de agua conectadas entre si. El llenado de estos estanques se produce según criterios de apertura y cierre del sistema de riego de los campos agricolas proximos, a través de dos derivaciones realizadas sobre el canal que vierte sus aguas en un pabellón de madera y en una plaza arbolada con naranjos. Una tubería para la conducción de alcohol de una antigua fábrica de azúcar ha sido reciclada como chorro de agua en este espacio. En el interior, una de las naves

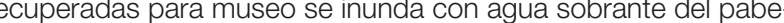
lón de madera. El circuito natural del agua se cierra en la plaza de naranjos donde se reconduce hasta el río Lanjarón para continuar su camino hacia el mar.

Plaza de agua y sombra. Reciclaje de un bosque de eucaliptus

El espacio situado delante del museo está formado por 17 naranjos de sombra y un suelo de troncos de madera de eucalipto inundado temporalmente por el agua de una acequia procedente de Sierra Nevada. Los troncos de madera empleados proceden de reciclados con parque caídos tras un vendaval de aire, cortados y la que emergen ordenadamente los naranjos, cambia de aspecto según el caudal de la acequia. En momentos de escaso caudal se convierte en un lugar accesible, mientras que con caudal abundante la plaza se inunda hasta convertirse en un espejo que refleja cuanto sucede alrededor La lámina de agua extendida sobre los 作 parecen flotar en la plaza.

\section{Participación y cohesión social}

as obras del museo han sido llevadas a cabo por una empresa del pueblo con la colaboración de personas del lugar implicadas con su patrimonio y paisaje. El jardinero municipal, a quien se debe la alfombra de madera de eucalipto del suelo, fue la persona encargada del reciclaje de los árboles caídos tras un vendaval que azotó durante días el parque natural. Y junto a este rabajo el de los componentes del estudio de arquitectura en que se realizó el proyecto, que se desplazaron a Lanjarón para que se realizo el proyecto, que se desplazaron a Lanjarón para levar a cabo las tareas de colocacion de los troncos de madera. La recuperación del antiguo molino aspira a convertirse de este modo en una acción participativa de la comunidad, un hito en la identidad cultural del municipio que favorezca el conocimiento del medio y de su historia y una mejora de la cohesión social. E proyecto encierra tambien la narracion de una historia mas amplia, como los 17 naranjos que forman la plaza, 17 historias sobre personas relacionadas con el pueblo de Lanjarón y el agua. El hecho de construir un museo "entre todos" ha permitido que la recuperación de este espacio se entienda como un patrimonio los niños a los ancianos, que contribuyen a la divulgación de una historia viva a los nuevos visitantes. 


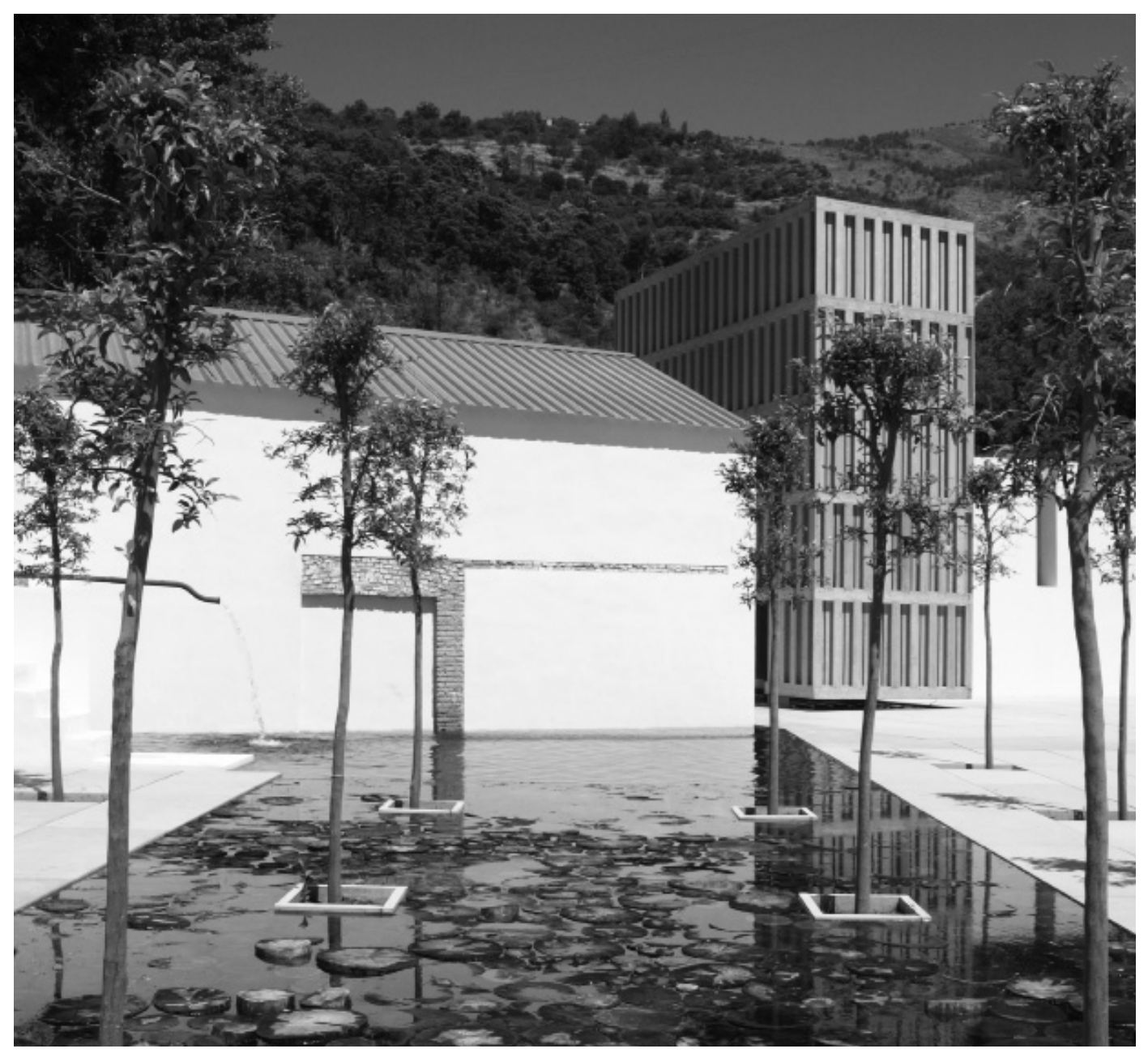

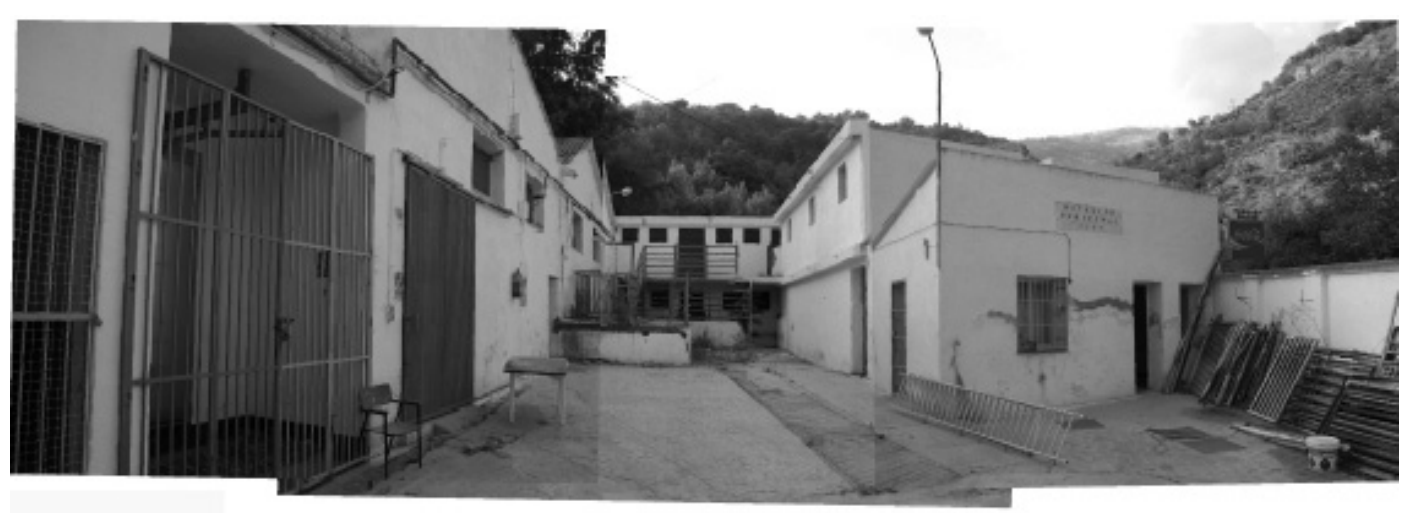

^ Estado inicial ๑ Juan Domingo Santos
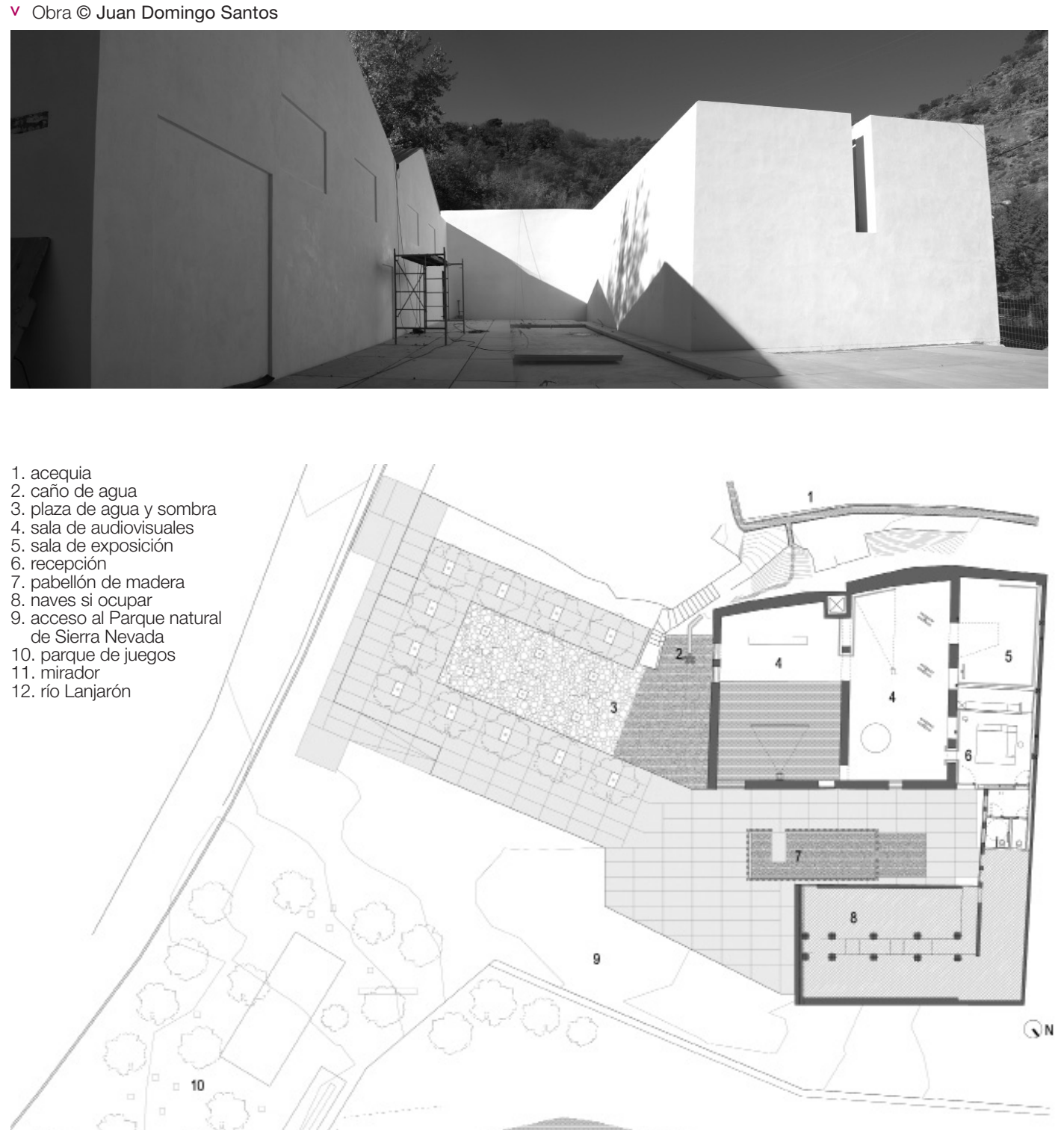

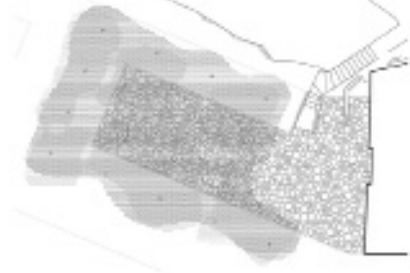

Espacio accesible (sin agua)

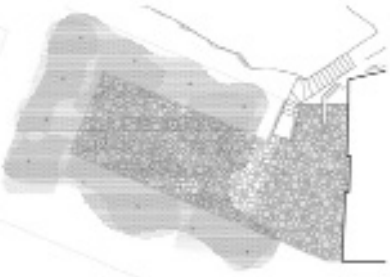

Fase 1. Plaza parcialmente accesible

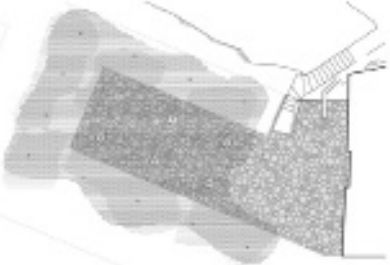

Fase 2. Plaza inundada
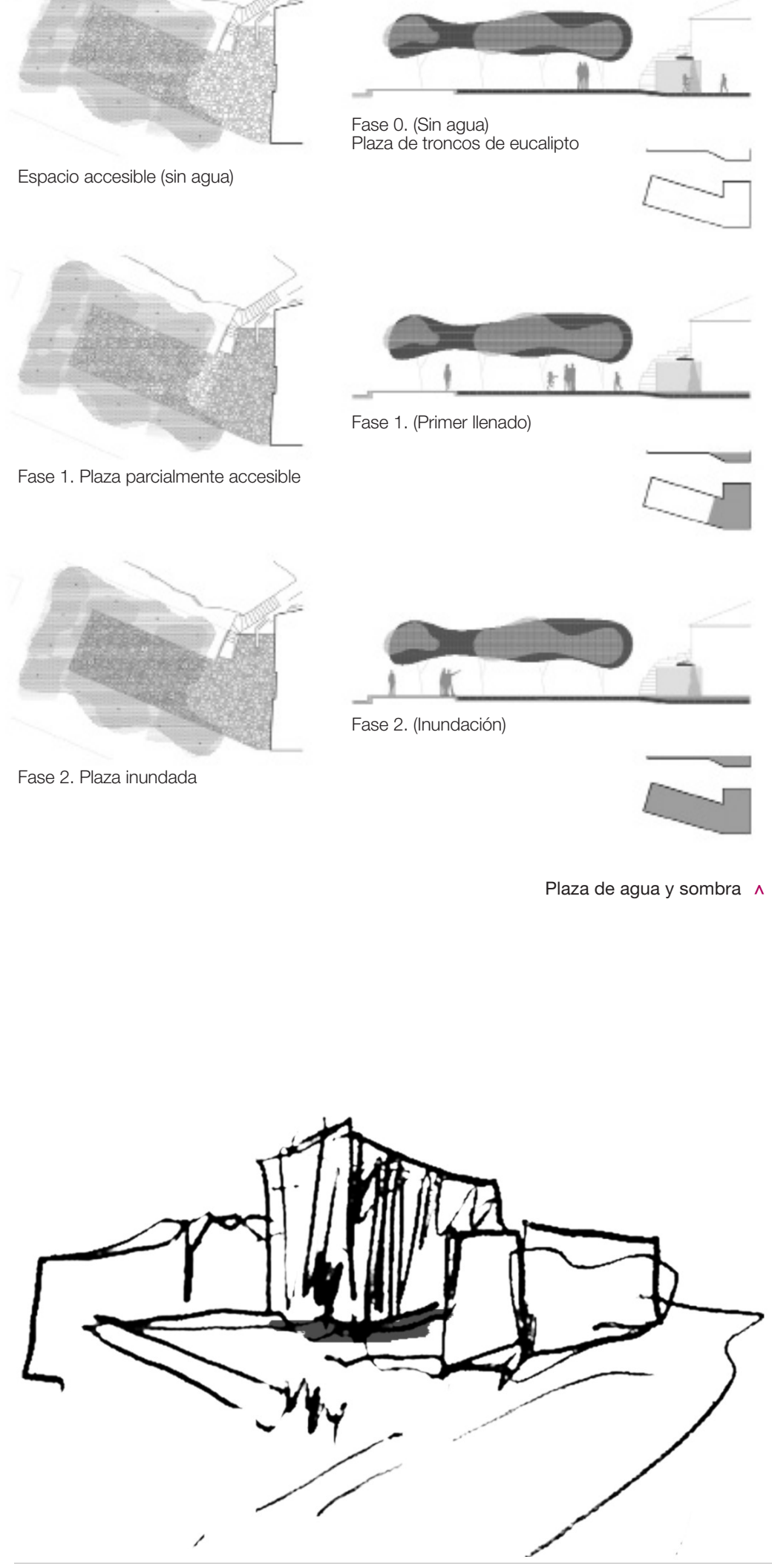

Plaza de agua y sombra $\wedge$

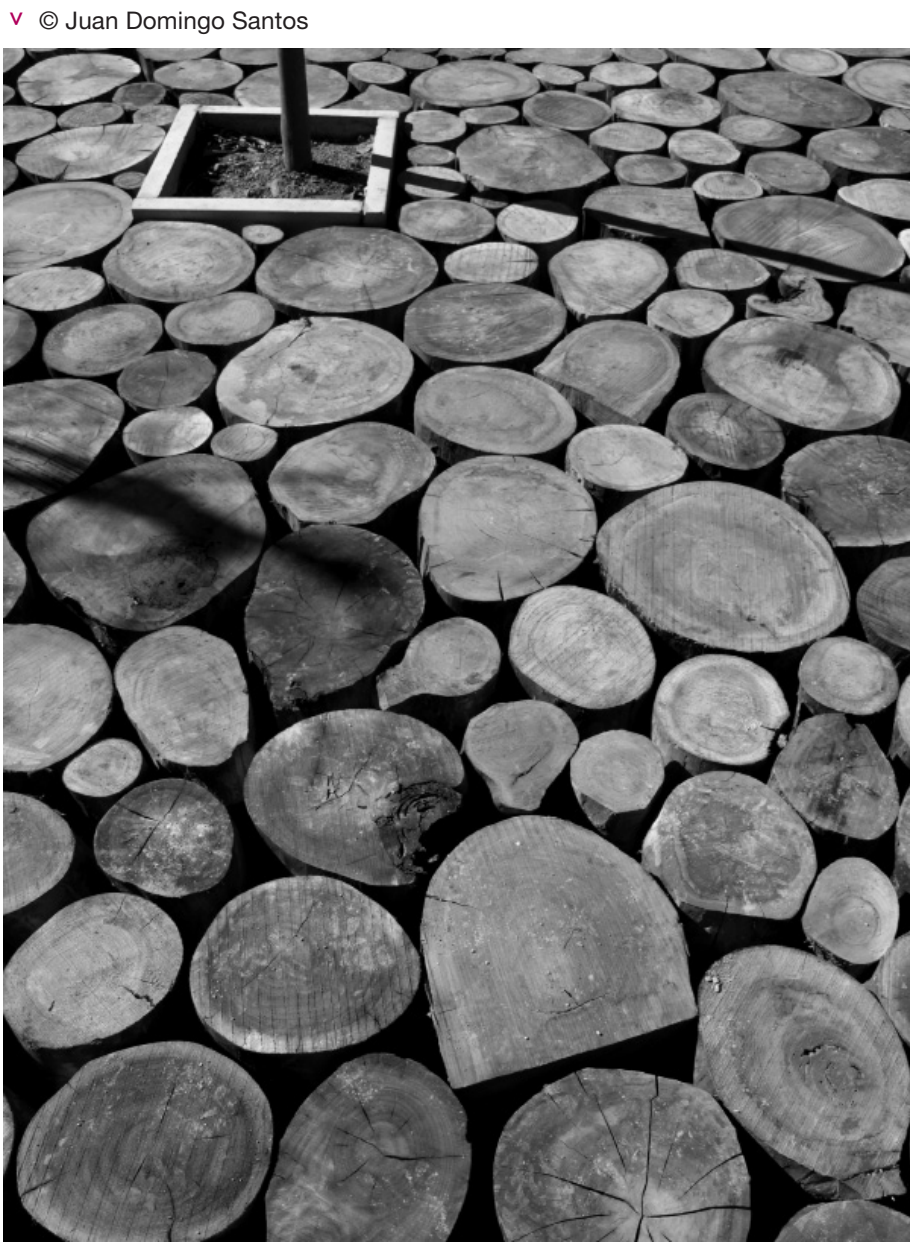

